\title{
Les lipides dans la maladie d'Alzheimer
}

\author{
Valérie Duflot ${ }^{*}$ \\ Reçu le 12 juillet 2018 - Accepté le 18 juillet 2018
}

Pathologie neurodégénérative progressive, la maladie d'Alzheimer est devenue un problème majeur de santé publique dans les pays occidentaux où l'espérance de vie s'est profondément allongée. Elle devrait toucher plus de 100 millions de personnes d'ici à 2050 , selon les estimations de la fédération internationale Alzheimer's disease International.

Bien que décrite dès 1906 par Alois Alzheimer, cette maladie n'aura été reconnue comme problème de santé publique que relativement récemment, comme le rappelle Jean-François Dartigues (Inserm U1219, Bordeaux, France) dans son article intitulé "Alzheimer's disease in the city », qui fait le point sur la prise en charge sociale et médicale de la maladie d'Alzheimer (Dartigues, 2018).

Malgré l'importante population touchée, aucun traitement curatif n'est encore disponible et seuls des traitements qui pourraient ralentir les symptômes sont parfois proposés. La prévention de cette maladie représente donc un défi majeur pour la recherche et la nutrition pourrait être un des facteurs clés des stratégies envisagées.

Comme le rappellent George Bloom et Andrés Norambuena (2018) dans leur article, la maladie d'Alzheimer se caractérise par l'accumulation dans le cerveau de deux types de structures anormales: des plaques amyloïdes extracellulaires et des enchevêtrements neurofibrillaires. Or, les oligomères amyloïde- $\beta$ seraient les principaux moteurs de la pathogenèse de la maladie: ils affameraient les neurones des malades, les rendant incapable d'utiliser efficacement les nutriments et facteurs trophiques qui les entourent.

Pour mettre à jour les mécanismes sous-jacents dans cette maladie, d'autres équipes travaillent sur l'information métabolique, à défaut de pouvoir analyser le cerveau in vivo. Leur objectif: démêler des mécanismes qui n'ont pas été pris en compte, tels que ceux reliant le cerveau au foie et à l'intestin ou à d'autres organes. Ainsi, Min Kim et Cristina Legido-Quigley (Institute of pharmaceutical science, King's College London, UK) signent dans ce dossier une revue des études relevant de ce champ et des voies moléculaires qui ont été signalées chez les modèles animaux et humains de la maladie d'Alzheimer (Kim et Legido-Quigley, 2018). Il en ressort qu'un grand nombre d'études ont utilisé la métabolomique afin d'identifier, soit des perturbations des voies métaboliques qui reflètent les changements associés à la pathologie, soit un panel de métabolites qui pourraient potentiellement être utilisés comme outils de diagnostic de la maladie d'Alzheimer. Ainsi, il apparaît que

\footnotetext{
* Correspondence : contact@ocl-journal.org
}

de multiples altérations métaboliques pourraient survenir au cours de l'évolution de cette maladie. Ceux-ci mettent en jeu les lipides, les métabolites impliqués dans les systèmes de neurotransmetteurs et le métabolisme énergétique : augmentation des céramides, appauvrissement des phospholipides et neurotransmetteurs, épuisement des stérols. L'homéostasie des lipides semble donc centrale dans la maladie d'Alzheimer, ce qui ne paraît guère surprenant puisque les principaux constituants du cerveau sont l'eau et les lipides.

$\mathrm{Au}$ rang des lipides majeurs étudiés, les acides gras polyinsaturés (AGPI). Au Canada, où 17 millions d'adultes âgés entre 30-64 ans pourraient bénéficier d'une intervention de prévention pour diminuer leur risque de développer la maladie d'Alzheimer, l'équipe de recherche de Mélanie Plourde (Research Center on Aging, Sherbrooke, Canada) travaille sur le métabolisme des AGPI n-3 à longue chaine (acides eicosapentaénoïque [EPA] et docosahexaénoïque [DHA]). En effet, chez les personnes génétiquement à risque (porteurs de l'allèle epsilon 4 de l'apolipoprotéine E, les cinétiques du DHA et de l'EPA sont modifiées avec l'âge, mais pas celle de l'AGPI à longue chaîne de la série n-6, l'acide arachidonique (ARA). En outre, chez les personnes âgées, la synthèse du DHA débute $2 \mathrm{~h}$ après la consommation d'EPA, contre 7 jours chez les participants jeunes. Pour Mélanie Plourde (2018), il est nécessaire de comprendre si les besoins en DHA des personnes âgées sont plus importants que ceux des jeunes (d'où sa synthèse précoce à partir de l'EPA) ou si la capacité des seniors à utiliser le DHA est plus basse (ce qui expliquerait son accumulation dans le plasma).

L'acide arachidonique, second acide gras polyinsaturé cérébral et premier de la série des n-6, est une cible de choix des recherches. En effet, les oligomères de peptides $\beta$ amyloïde sont désormais reconnus comme l'agent principal de la maladie d'Alzheimer, bien que la présence de la protéine tau soit nécessaire à leur action. Dans la revue qu'ils publient dans ce dossier, Katleen Pinchaud, Katy Maguin-Gaté et Jean-Luc Olivier (université de Lorraine, Vandœuvre-Lès-Nancy, France) rappellent avoir établi, avec d'autres auteurs, que la phospholipase A2 cytosolique, spécifique de l'acide arachidonique, assure les effets neurotoxiques des oligomères de peptide $\beta$ amyloïde, et qu'un régime riche en acide arachidonique augmente la sensibilité des souris aux effets de ces oligomères, sans augmentation majeure de ses niveaux cérébraux. Ceci suggère que cet acide gras peut agir sur le cerveau par des effets périphériques comme une sub-inflammation dont le rôle dans la relation intestin-cerveau est discuté dans la littérature (Pinchaud et al., 2018). Des études supplémentaires demeurent néanmoins 
nécessaires sur les divers mécanismes induits par des apports alimentaires élevés, y compris la modulation de l'inflammation, la modification du microbiote intestinal, ainsi que l'influence sur les barrières intestinales et sanguines.

Le cholestérol, constituant majeur des membranes cellulaires, est également une cible de choix. Marie-Claude Potier, Linda Hanbouch et Catherine Marquer (Institut du cerveau et de la moelle épinière, hôpital de la Pitié-Salpêtrière, Paris, France) passent en revue les preuves suggérant que l'augmentation transitoire des niveaux de cholestérol de la membrane des neurones affectera profondément le positionnement transmembranaire de la protéine précurseur amyloïde et son traitement, entraînant une surproduction de peptides amyloïdes- $\beta$ (Potier et al., 2018).

Autre axe de recherche: l'acide phosphatidique, qui joue un rôle particulier et central dans diverses fonctions cellulaires essentielles. L'équipe de Nicolas Vitale (Institut des neurosciences cellulaires et intégratives, Strasbourg, France) résume dans son article les observations récentes qui illustrent le fait que l'acide phosphatidique, produit par deux voies enzymatiques distinctes impliquant les phospholipases D et les diacylglycérol-kinases, est impliqué dans le développement neuronal et la neurosécrétion. Pour finir, ils présentent des résultats d'analyses lipidomiques qui indiquent que différentes formes de l'acide phosphatidique sont produites au cours de ces deux processus neuronaux majeurs (Tanguy et al., 2018).

De son côté, Stephen Cunnane (université de Sherbrooke, Canada), récipiendaire de la médaille Chevreul 2017, considère que l'impact du Yin et Yang dans l'équilibre cérébral n'est nulle-part ailleurs plus évident que dans la maladie d'Alzheimer (Cunnane, 2018). Une des raisons pour lesquelles les essais de traitement de cette pathologie ont tous échoué pourrait être, selon lui, que l'apport insuffisant de glucose dans certaines régions du cerveau (de l'ordre de $-10 \%$ ) précède le déficit cognitif chez les personnes à risques. Ainsi, seuls des traitements susceptibles de restaurer l'apport énergétique via les cétones pourraient potentiellement s'avérer efficaces. Par analogie aux cétones permettant l'économie de glucose dans le métabolisme énergétique du cerveau, il est possible que le précurseur des AGPI n-3, l'acide $\alpha$-linolénique (ALA) et l'EPA (qui sont également captés par le cerveau) permettent de préserver la fonction structurelle du DHA. Ainsi, loin d'être un métabolisme futile, le transport d'ALA et d'EPA vers le cerveau pour être bêta-oxydés pourrait participer à une économie de DHA et à la production de cétones.

À noter que, dans ce même numéro d'OCL, Didier Majou (ACTIA, Paris, France) propose un article sur l'évolution du cerveau de l'homme et les rôles clés du DHA et du gène de la $\Delta 6$-désaturase (Majou, 2018). Il y explique qu'avec l'apport de la cuisson et de nouvelles mutations plus productives de FADS2, le volume cérébral a trouvé son apogée chez Homo neanderthalensis dans un écosystème alimentaire favorable en acide $\alpha$-linolénique et DHA. Mais, la glaciation de Würm bouleversa cet équilibre et fit apparaître sa fragilité (cerveau, fertilité). Homo sapiens, avec l'avantage de nouveaux variants du gène $F A D S 2$, s'adapta à cet environnement rigoureux, alors que l'Homme de Néandertal ne put le faire et disparut.
Enfin, à la fin de ce numéro, Michael Crawford (Institute of brain chemistry and human nutrition, and Department of cancer and surgery, Chelsea and Westminster Hospital Campus of Imperial College, London, Royaume-Uni), récipiendaire de la médaille Chevreul 2015, co-signe deux articles (Crawford et al., 2018a, 2018b) relativement révolutionnaires sur le rôle des électrons $\pi$ de l'acide docosahexaénoïque dans la fonction cérébrale. Il y soulève trois problèmes majeurs dans les théories basées sur le rôle des protéines neuronales : le rôle principal des protéines dans le cerveau est de fournir la quantité phénoménale d'énergie nécessaire; la principale composante structurelle du cerveau est le lipide et non la protéine; et il n'existe aucune explication à la précision presque absolue requise pour l'acuité visuelle et la fonction de mémoire dans les théories basées sur les protéines. Pourtant, cette précision absolue est essentielle. Aussi, il propose une thèse basée sur les propriétés mécanistiques quantiques spécifiques des électrons $\pi$ des lipides, qui pourraient fournir la précision requise pour la photoréception, la mémoire et la conscience.

\section{Références}

Bloom GS, Norambuena A. 2018. Alzheimer's disease as a metabolic disorder. OCL 25(4): D403. https://doi.org/10.1051/oc1/2018041.

Crawford MA, Thabet M, Wang Y. 2018a. An introduction to a theory on the role of $\pi$-electrons of docosahexaenoic acid in brain function. The Quantum Brain. OCL 25(4): A402. Available from https://doi.org/10.1051/ocl/2018010.

Crawford MA, Thabet M, Wang Y, Broadhurst CL, Schmidt WF. $2018 \mathrm{~b}$. A theory on the role of $\pi$-electrons of docosahexaenoic acid in brain function. The six methylene-interrupted double bonds and the precision of neural signaling. OCL 25(4): A403. Available from https://doi.org/10.1051/ocl/2018011.

Cunnane SC. 2018. Ketones, omega-3 fatty acids and the Yin-Yang balance in the brain: insights from infant development and Alzheimer's disease, and implications for human brain evolution. OCL 25(4): D409. Available from https://doi.org/10.1051/ocl/2018020.

Dartigues J-F. 2018. Alzheimer in the City. OCL 25(4): D402. Available from https://doi.org/10.1051/ocl/2018037.

Kim M, Legido-Quigley C. 2018. Small molecule biomarkers in Alzheimer's disease. OCL 25(4): D404. Available from https:// doi.org/10.1051/oc1/2018027.

Majou D. 2018. Evolution of the Human Brain: the key roles of DHA (omega-3 fatty acid) and $\triangle 6$-desaturase gene. OCL 25(4): A401. Available from https://doi.org/10.1051/ocl/2017059.

Pinchaud K, Maguin-Gaté K, Olivier J-L. 2018. Dietary arachidonic acid: a Janus face actor in brain and Alzheimer's disease? OCL 25 (4): D406. Available from https://doi.org/10.1051/oc1/2018033.

Plourde M. 2018. Aging, cognitive decline, apolipoprotein E and docosahexaenoic acid metabolism. OCL 25(4): D405. Available from https://doi.org/10.1051/ocl/2018032.

Potier M-C, Hanbouch L, Marquer C. 2018. Cholesterol and ApoE in Alzheimer's disease. OCL 25(4): D407.

Tanguy E, Wang Q, Coste de Bagneaux P, Fouillen L, Thahouly T, Ammar M-R, et al. 2018. Different species of phosphatidic acid are produced during neuronal growth and neurosecretion. OCL 25 (4): D408. Available from https://doi.org/10.1051/oc1/2018024.

Citation de l'article : Duflot V. 2018. Les lipides dans la maladie d'Alzheimer. OCL 25(4): D401. 